\title{
Novel serum biomarkers modified by the body mass index z-score for the detection of liver fibrosis and steatosis in children with chronic hepatitis $C$
}

\author{
Maria Pokorska-Śpiewak ${ }^{1,2^{*}}$ D, Barbara Kowalik-Mikołajewska ${ }^{1,2}$, Małgorzata Aniszewska ${ }^{1,2}$, Magdalena Pluta ${ }^{1,2}$ \\ and Magdalena Marczyńska ${ }^{1,2}$
}

\begin{abstract}
Background: There is a need for validation of noninvasive alternatives to liver biopsy for the evaluation of fibrosis in children with chronic hepatitis $\mathrm{C}(\mathrm{CHC})$. The aim of this study was to evaluate the diagnostic performance of serum biomarkers modified by the body mass index z-score (BMI z-score) for the detection of fibrosis and steatosis in children with $\mathrm{CHC}$.
\end{abstract}

Methods: Thirty children aged $9.4 \pm 3.7$ years (14 males, 16 females) with CHC underwent liver biopsy. Fibrosis was scored using a 5-point METAVIR scale ( $\geq 2$ = significant fibrosis). For all the children, the following noninvasive markers were calculated: The aspartate transaminase (AST)-to-platelets ratio index (APRI), the modified APRI (M-APRI: BMI z-score $\times$ APRI), the Fibrosis-4 index (FIB-4), the modified FIB-4 (M-FIB-4: BMI z-score $\times$ FIB-4), and a novel marker, B-AST (BMI z-score $\times$ AST). The area under the receiver operator characteristic curve (AUROC) was calculated to detect significant fibrosis and steatosis.

Results: In the histopathological evaluation, 22/30 (73\%) patients presented with fibrosis, and 8/30 (27\%) presented with steatosis. For the detection of significant fibrosis, the AUROCs for M-APRI, M-FIB-4 and B-AST were 0.842, 0.823, and 0.848 , respectively. For significant steatosis, the AUROCs were more than 0.9 for all markers that included the BMI z-score. B-AST, with a cut-off of 92.8 , showed $71 \%$ sensitivity and $95 \%$ specificity for detecting significant fibrosis. For predicting severe steatosis, B-AST had 100\% sensitivity and $92 \%$ specificity. Negative values of all three markers that included BMI z-scores excluded all patients with both significant fibrosis and significant steatosis.

Conclusions: Including the BMI z-score in serum biomarker formulas enhances their diagnostic ability to detect significant fibrosis and steatosis. B-AST may thus act as an effective alternative to liver biopsy.

Keywords: Hepatitis C, Liver biopsy, Liver fibrosis, Liver steatosis

\section{Background}

Liver biopsy has been considered a standard method for evaluating liver fibrosis and steatosis in children with chronic hepatitis $\mathrm{C}(\mathrm{CHC})$ [1-3]. However, it has several limitations: it is invasive and painful, has inter- and intraobserver variability, and is prone to sampling errors $[1,3]$. Therefore, efforts have been made to develop

\footnotetext{
* Correspondence: mpspiewak@gmail.com

${ }^{1}$ Department of Children's Infectious Diseases, Medical University of Warsaw, ul. Wolska 37, 01-201 Warsaw, Poland

${ }^{2}$ Hospital of Infectious Diseases, ul. Wolska 37, 01-201 Warsaw, Poland
}

alternative, noninvasive methods to liver biopsy, including imaging studies (elastography) and serum biomarkers $[3,4]$. According to the recent recommendations of the European Association for the Study of the Liver (EASL), noninvasive methods can be used instead of liver biopsy to assess liver disease severity prior to antiretroviral therapy, and liver biopsy should be reserved only for cases with potential additional comorbidities or any uncertainty [2]. Many of these noninvasive methods have been evaluated in adults with $\mathrm{CHC}$ for their ability to determine fibrosis [5]. However, data on the accuracy of 
noninvasive tests in pediatric patients with $\mathrm{HCV}$ are limited, and none of these methods has been fully validated in children to date $[3,4]$.

The serum biomarkers include both direct and indirect markers. Direct markers (e.g., glycoproteins, collagens, collagenases, and metalloproteases) reflect the removal or deposition of extracellular matrix in the liver [5]. Indirect markers can be identified in routine blood tests and indicate alterations in liver function [5]. Such markers include the aspartate transaminase-to-platelets ratio index (APRI) and the Fibrosis-4 index (FIB-4), which have been validated to predict significant fibrosis and cirrhosis in adult patients with chronic HCV infection [5-7]. Simple serum biomarkers are widely available, inexpensive, and easy to calculate and therefore provide a desirable alternative to liver biopsy [8].

In our recent studies, we have shown that in children with $\mathrm{CHC}$, both fibrosis and steatosis were positively associated with the body mass index z-score (BMI z-score) $[9,10]$. Thus, the aim of the present study was to analyze the diagnostic performance of APRI and FIB-4 to determine liver fibrosis and steatosis in children with CHC. Additionally, the diagnostic performance of these biomarkers when modified by including the BMI z-score in the formulas was established, and B-AST, a novel simple marker based on the BMI z-score and aspartate aminotransferase (AST) only, was proposed, and its diagnostic performance was analyzed.

\section{Methods}

\section{Patients}

This prospective clinicopathological study comprised consecutive treatment-naïve patients chronically infected with $\mathrm{HCV}$ who underwent a liver biopsy in our tertiary health care department between 2010 and 2014. Noninvasive serum biomarker analysis was performed simultaneously with liver biopsy as part of the qualification procedure for the antiviral treatment, according to the current European recommendations [2]. In addition, liver biopsy was required by the National Health Found for every patient prior to inclusion to the therapeutic program. The $\mathrm{CHC}$ diagnosis was established in patients with at least a 6-month history of hepatitis based on elevated alanine and aspartate aminotransferase (ALT and AST) serum levels and positive anti-HCV testing and was confirmed with nucleic acid testing/positive $\mathrm{HCV}$ RNA real-time polymerase chain reaction analysis (RTPCR method; Amplicor, Roche; Cobas TaqMan, Roche). Both biochemical and serological testing were performed using commercially available laboratory kits (Vitros 5600, Ortho-Clinical Diagnostics, Johnson \& Johnson). The upper limits of normal (ULN) for ALT and AST were established as $40 \mathrm{IU} / \mathrm{l}$. The most likely route and time of infection were established using available medical records. The putative duration of infection was calculated from the beginning of the risk exposure.

Patients with hepatitis B virus, human immunodeficiency virus infection, autoimmune hepatitis, nonalcoholic fatty liver disease, Wilson's disease, or alpha 1antitrypsin deficiency were excluded from this study. The patients' weights and heights were recorded on the day of the biomarker examination. BMI z-scores were calculated using the World Health Organization (WHO) Child Growth Standards and Growth Reference data with the WHO anthropometric calculator, AnthroPlus v.1.0.4.

\section{Biomarker evaluation}

The biomarker determinations were performed using commercially available laboratory kits (XT-1800i, Sysmex for platelets; Vitros 5600, Ortho-Clinical Diagnostics, Johnson \& Johnson for biochemical parameters). The noninvasive serum biomarker analysis included APRI and FIB-4, which were calculated according to the published analytic recommendations [6, 7], as follows:

$$
\begin{aligned}
\text { APRI }= & {\left[\text { AST }(\mathrm{IU} / \mathrm{L}) / \operatorname{AST} \mathrm{ULN}(\mathrm{IU} / \mathrm{L}) / \text { platelet } \operatorname{count}\left(10^{9} / \mathrm{L}\right)\right] } \\
& \times 100 ; \\
\text { FIB-4 }= & {[\operatorname{age}(\text { years }) \times \operatorname{AST}(\mathrm{IU} / \mathrm{L})] /\left[\text { platelet } \operatorname{count}\left(10^{9} / \mathrm{L}\right)\right.} \\
& \times \sqrt{\operatorname{ALT}(\mathrm{IU} / \mathrm{L})}]
\end{aligned}
$$

Additionally, modified APRI (M-APRI) and modified FIB-4 (M-FIB-4) were calculated by including BMI zscores in both formulas, as follows:

$$
\begin{aligned}
\mathrm{M}-\mathrm{APRI} & =\mathrm{BMI} z \text {-score } \times \text { APRI } ; \text { M-FIB- } 4 \\
& =\text { BMI z-score } \times \text { FIB- } 4 .
\end{aligned}
$$

A novel simple biomarker, B-AST $=$ BMI z-score $\times$ AST (IU/L), was also proposed and calculated.

\section{Histopathological evaluation}

Liver biopsy was performed percutaneously using a Menghini needle (Hepafix kit 1.4 or $1.6 \mathrm{~mm}$, Braun). An experienced pathologist who was unaware of the clinical data performed the histopathological evaluation. Fibrosis staging was evaluated using the METAVIR scoring system on a 5-point scale (F0 - no fibrosis; F1 - portal fibrosis without septa; F2 - portal fibrosis with few septa; F3 - numerous septa without cirrhosis; and F4 cirrhosis) [11]. Fibrosis was considered significant if the METAVIR F score was $\geq 2$. Liver steatosis was determined semi-quantitatively according to the percentage of hepatocytes containing fat droplets and was staged as follows: 0 - no steatosis; $1-$ minimal $(\leq 5 \%$ hepatocytes affected); 2 - mild (6-33\%); 3 - moderate (34-66\%); and $4-$ severe $(>66 \%)$. Steatosis was considered significant if more than $33 \%$ of the hepatocytes were affected (steatosis score $>2$ ). 


\section{Statistical analysis}

Continuous variables were tested for normal distribution using the Kolmogorov-Smirnov test and are expressed as the mean \pm standard deviations (SD) or as medians with interquartile ranges (IQR), as required. To analyze associations between the results of the five serum biomarkers and fibrosis and steatosis stages, Spearman correlation coefficients were obtained. A two-sided $p$ value $<0.05$ was considered significant.

Receiver operating characteristic (ROC) curves and areas under the ROC curve (AUROC) calculations were performed to assess the diagnostic performances of the noninvasive tests for identifying patients with any or significant liver fibrosis and steatosis, using liver biopsy as a reference standard. An analyzed method is considered perfect when the AUROC is $100 \%$, excellent when the AUROC is over $90 \%$, and good when the AUROC is over $80 \%$ [3,5]. Optimal cutoffs, sensitivity, specificity, and positive and negative predictive values were also calculated for each noninvasive test.

All statistical analyses were performed using the licensed MedCalc Statistical Software, ver. 17.2 (MedCalc, Mariakerke, Belgium).

\section{Ethical statement}

The investigation was concordant with the principles outlined in the Declaration of Helsinki and its amendments. Written informed consent was collected from all the patients and/or their parents/guardians before their inclusion in the study.

\section{Results}

\section{Patient characteristics}

Thirty children (14 male and 16 female) aged $9.4 \pm 3.7$ years with a mean $\mathrm{HCV}$ infection duration of $8.2 \pm 3.1$ years were included in this study. Most of the children (73\%) were infected vertically by an infected mother. The predominant $\mathrm{HCV}$ genotype in this group was 1 b. In $2 / 30(7 \%)$ cases, the BMI zscore indicated obesity ( $>2 \mathrm{SD}$ ). The clinical and laboratory characteristics of the study group are presented in Table 1.

\section{Histopathological evaluation}

Histopathological evaluation of the liver biopsy specimens revealed a mild stage of fibrosis in most cases: $22 / 30(73 \%)$ patients presented with some stage of fibrosis, and in $7 / 30$ (23\%) patients, the fibrosis was significant (METAVIR F score $\geq 2$ points). No cirrhosis cases were observed. Liver steatosis was detected in $8 / 30$ (27\%) patients, and in $4 / 30$ (13\%) patients, the steatosis was significant (> 33\% of hepatocytes affected, Table 1).
Table 1 Clinical and laboratory characteristics of the study group

\begin{tabular}{|c|c|c|}
\hline Characteristics & & Data \\
\hline Number & & 30 \\
\hline Sex & Male (\%)/female (\%) & $14(47) / 16(53)$ \\
\hline Age at liver biopsy (years) & Mean \pm SD & $9.4 \pm 3.7$ \\
\hline Duration of infection (years) & Mean \pm SD & $8.2 \pm 3.1$ \\
\hline BMI z-score & Mean \pm SD & $0.39 \pm 1.04$ \\
\hline Mode of infection & Vertical (\%) & $22(73)$ \\
\hline & Nosocomial (\%) & $7(23)$ \\
\hline HCV genotype (\%) & $1 \mathrm{a}$ & $2(7)$ \\
\hline & $1 b$ & $19(63)$ \\
\hline & 3 & $5(17)$ \\
\hline & 4 & $4(13)$ \\
\hline Viral load (IU/ml) & HCV median (IQR) & $5.95 \times 10^{5}\left(2.25 \times 10^{5}-1.53 \times 10^{6}\right)$ \\
\hline Laboratory findings & $\operatorname{ALT}(I U / I)$ & $60.5(36-79)$ \\
\hline Mean \pm SD/median (IQR) & AST (IU/I) & $50(37-68)$ \\
\hline & Platelets $\left(\times 10^{9} / \mathrm{l}\right)$ & $308.2 \pm 90.5$ \\
\hline METAVIR F & 0 & $8(27)$ \\
\hline & 1 & $15(50)$ \\
\hline & 2 & $7(23)$ \\
\hline & $3-4$ & 0 \\
\hline Steatosis & Any (\%) & $8(27)$ \\
\hline & Significant (\%) & $4(13)$ \\
\hline APRI & Mean \pm SD & $0.48 \pm 0.26$ \\
\hline FIB-4 & Mean \pm SD & $0.22 \pm 0.13$ \\
\hline M-APRI & Mean $\pm S D$ & $0.28 \pm 0.69$ \\
\hline M-FIB-4 & Mean $\pm S D$ & $0.09 \pm 0.28$ \\
\hline B-AST & Mean \pm SD & $31.71 \pm 69.87$ \\
\hline
\end{tabular}

ALT alanine aminotransferase, $A S T$ aspartate aminotransferase, $B M I$ body mass index, APRI aspartate-to-platelet Ratio Index, FIB-4 Fibrosis-4 test, M-APRI modified aspartate-to-platelet ratio index (BMI z-score x APRI); M-FIB-4 modified Fibrosis-4 index (BMI z-score x FIB-4), B-AST BMI z-score x AST

\section{Noninvasive evaluation}

Five noninvasive serum biomarkers were calculated according to the formulas described above, and the mean values for each test are presented in Table 1 . The APRI exceeded 0.7 in 6/30 (20\%) cases, and in 3/30 (10\%) patients, the APRI was greater than 1.0. The maximal FIB4 value was 0.57 , and it did not exceed 1.0 in any case.

\section{Relationship between noninvasive and histopathological evaluations}

Positive associations between the fibrosis stages determined by the histopathological evaluation and the MAPRI and B-AST scores were revealed $(p=0.01$ and $p=0.001$, respectively, Table 2 ). A trend toward such an association was observed for APRI and M-FIB-4 ( $p=0.06$ and $p=0.05$, respectively, Table 2$)$. Steatosis stages were positively associated with the APRI, MAPRI, and M-FIB- 4 scores ( $p=0.02$ for all evaluations), and they trended toward an association with the B-AST score $(p=0.08$, Table 2$)$. 
Table 2 Association between the five biomarkers and the stage of fibrosis (METAVIR F score) and between the biomarkers and the presence of steatosis

\begin{tabular}{lll}
\hline Marker & METAVIR F & Steatosis \\
\hline APRI & $0.34(-0.03-0.63), p=0.06$ & $0.42(0.08-0.68), p=0.02$ \\
M-APRI & $0.44(0.08-0.69), p=0.01$ & $0.40(0.05-0.67), p=0.02$ \\
FIB-4 & $0.18(-0.20-0.51), p=0.35$ & $0.09(-0.27-0.44), p=0.61$ \\
M-FIB-4 & $0.36(-0.01-0.64), p=0.05$ & $0.40(0.04-0.66), p=0.02$ \\
B-AST & $0.56(0.24-0.77), p=0.001$ & $0.32(-0.05-0.61), p=0.08$ \\
\hline
\end{tabular}

Data are presented as correlation coefficients (95\% confidence intervals) and $p$-values

Figure 1 shows the diagnostic performance (ROC curves) of the five analyzed noninvasive tests for all fibrosis diagnoses (Fig. 1a) and for significant fibrosis diagnoses (METAVIR $F \geq 2$ points, Fig. 1b). For the diagnosis of any fibrosis stage, the corresponding AUROCs were below 0.8 for all tests, and the highest AUROC value was observed for APRI: 0.719 (0.5260.867) (Fig. 1a). In the significant fibrosis cases, the AUROCs for all three tests that included BMI z-scores exceeded 0.8 (0.842, 0.823, and 0.848 for the M-APRI, M-FIB-4, and B-AST, respectively), whereas the AUROCs for the APRI and FIB-4 were below 0.8 (Table 3). The novel marker, B-AST, predicted significant fibrosis with a cut-off of 92.8 with $71.4 \%$ sensitivity and $95.7 \%$ specificity.

The diagnostic performances of the five noninvasive tests for determining steatosis are presented in Fig. 2 and Table 4. For the detection of any steatosis stage, the AUROC was highest for the APRI (0.768). The AUROCs for the other tests did not exceed 0.7 (Fig. 2a). The diagnostic value of the tests for detecting significant steatosis was excellent (AUROC >0.9) for all three tests that included the BMI z-scores (0.923, 0.942, and 0.942 for MAPRI, M-FIB-4, and B-AST, respectively). For the APRI, the AUROC was good (0.837); however, for the FIB-4, the AUROC was insufficient (0.683). B-AST, with a cutoff of 92.8, predicted significant steatosis with $100 \%$ sensitivity and $92.3 \%$ specificity (Table 4 ).

\section{Discussion}

In the era of direct-acting antivirals (DAAs), successful treatment of $\mathrm{CHC}$ is achievable for nearly all infected patients, and DAAs are recommended for all adults with chronic HCV infection [2, 12]. The availability of DAAs for pediatric patients with $\mathrm{CHC}$ is expected within the coming months. According to the current recommendations, histopathological evaluation of liver disease may not be necessary for all patients prior to therapy. However, the majority of patients require testing to determine the stage of fibrosis, which is vital for determining the urgency of treatment, the duration of treatment in
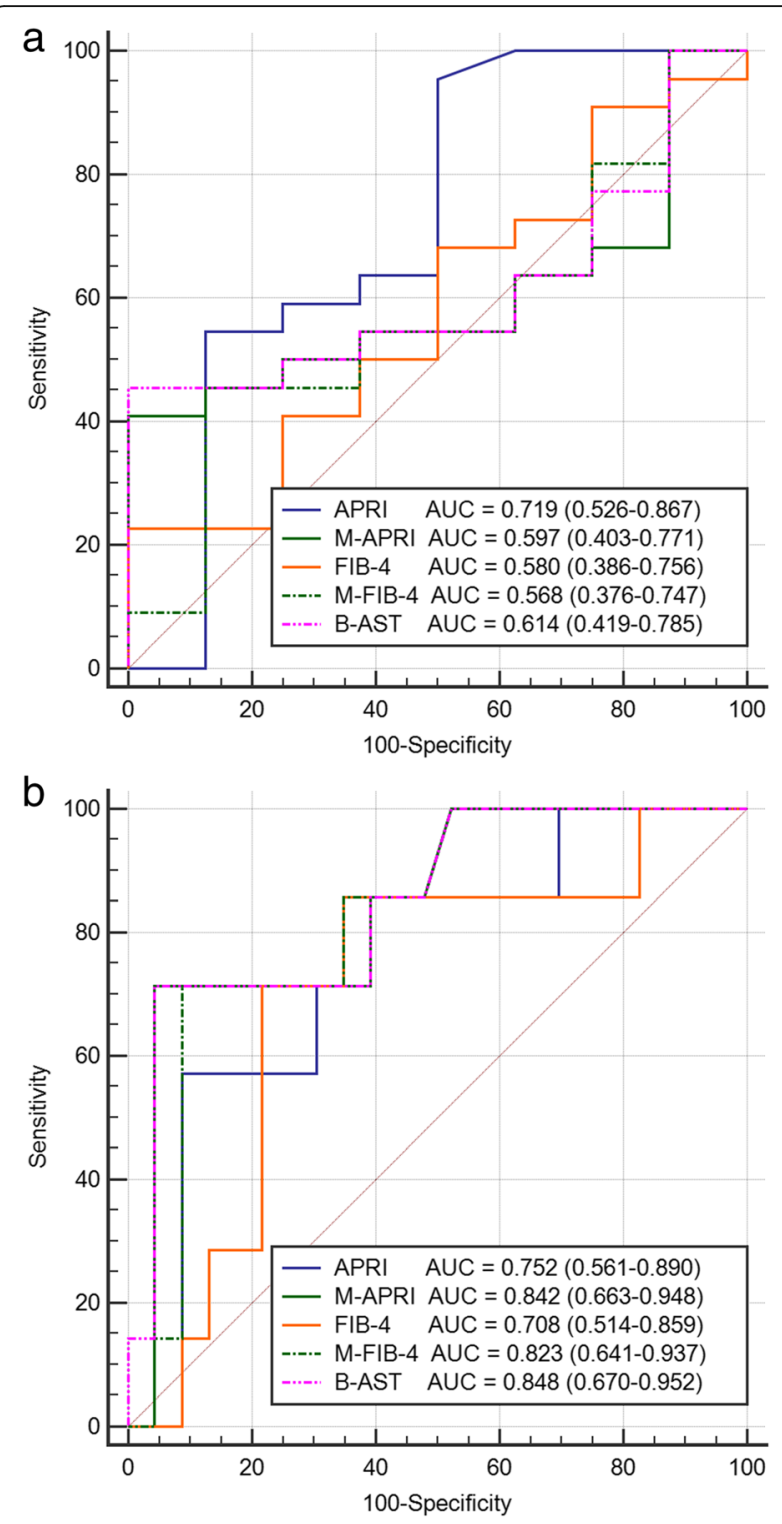

Fig. 1 Receiver operating characteristic (ROC) curves of the five noninvasive liver fibrosis tests for detecting fibrosis. a for fibrosis at any stage (METAVIR F score $>0$ points). $\mathbf{b}$ for significant fibrosis (METAVIR F score $\geq 2$ points)

some instances, and the need for more intensive clinical monitoring [12].

Several noninvasive markers of liver fibrosis based on a combination of different biochemical parameters have been developed recently and analyzed in adult patients with $\mathrm{CHC}[5,7,8,13]$. A meta-analysis of 40 studies revealed that an APRI score greater than 1.0 had a sensitivity of $76 \%$ and specificity of $72 \%$ for predicting cirrhosis. Additionally, APRI scores greater than 0.7 had a sensitivity of $77 \%$ and specificity of $72 \%$ for predicting significant hepatic fibrosis [13]. In a study by Sterling et al., FIB-4 scores $<1.45$ had a negative predictive value of 
Table 3 Diagnostic performance of five non-invasive tests for determining significant fibrosis (METAVIR F $\geq 2$ )

\begin{tabular}{llllll}
\hline \multicolumn{2}{l}{ Significant fibrosis (METAVIR F $\geq 2)$} & & & & \\
\hline Test & APRI & M-APRI & FIB-4 & M-FIB-4 & B-AST \\
\hline AUROC (95\% Cl) & $0.752(0.561-0.890)$ & $0.842(0.663-0.948)$ & $0.708(0.514-0.859)$ & $0.823(0.641-0.937)$ & $0.848(0.670-0.952)$ \\
Cut-off & 0.656 & 0.577 & 0.180 & 0.179 & 92.82 \\
Sensitivity (95\% Cl) & $57.1(18.4-90.1)$ & $71.4(29.0-96.3)$ & $85.7(42.1-99.6)$ & $71.4(0.29-96.3)$ & $71.4(0.29-96.3)$ \\
Specificity (95\% Cl) & $91.3(72.0-98.9)$ & $95.6(78.1-99.9)$ & $65.2(42.7-83.6)$ & $91.30(72.0-98.9)$ & $95.7(78.1-99.0)$ \\
+ PV & $66.7(31.5-89.7)$ & $83.3(41.0-97.3)$ & $42.9(28.4-58.6)$ & $71.4(38.0-91.1)$ & $83.3(41.0-97.3)$ \\
- PV & $87.5(74.7-94.3)$ & $91.7(77.3-97.3)$ & $93.7(70.5-99.0)$ & $91.3(76.4-97.2)$ & $91.7(77.3-97.3)$ \\
\hline
\end{tabular}

APRI aspartate-to-platelet ratio index, FIB-4 Fibrosis-4 index, M-APRI modified aspartate-to-platelet ratio index (BMI z-score x APRI), M-FIB-4 modified Fibrosis-4 index (BMI z-score x FIB-4), B-AST BMI z-score x AST, AUROC area under the receiver operating characteristic, $95 \% \mathrm{Cl} 95 \%$ confidence interval, $+P V$ positive predictive value, $-P V$ negative predictive value

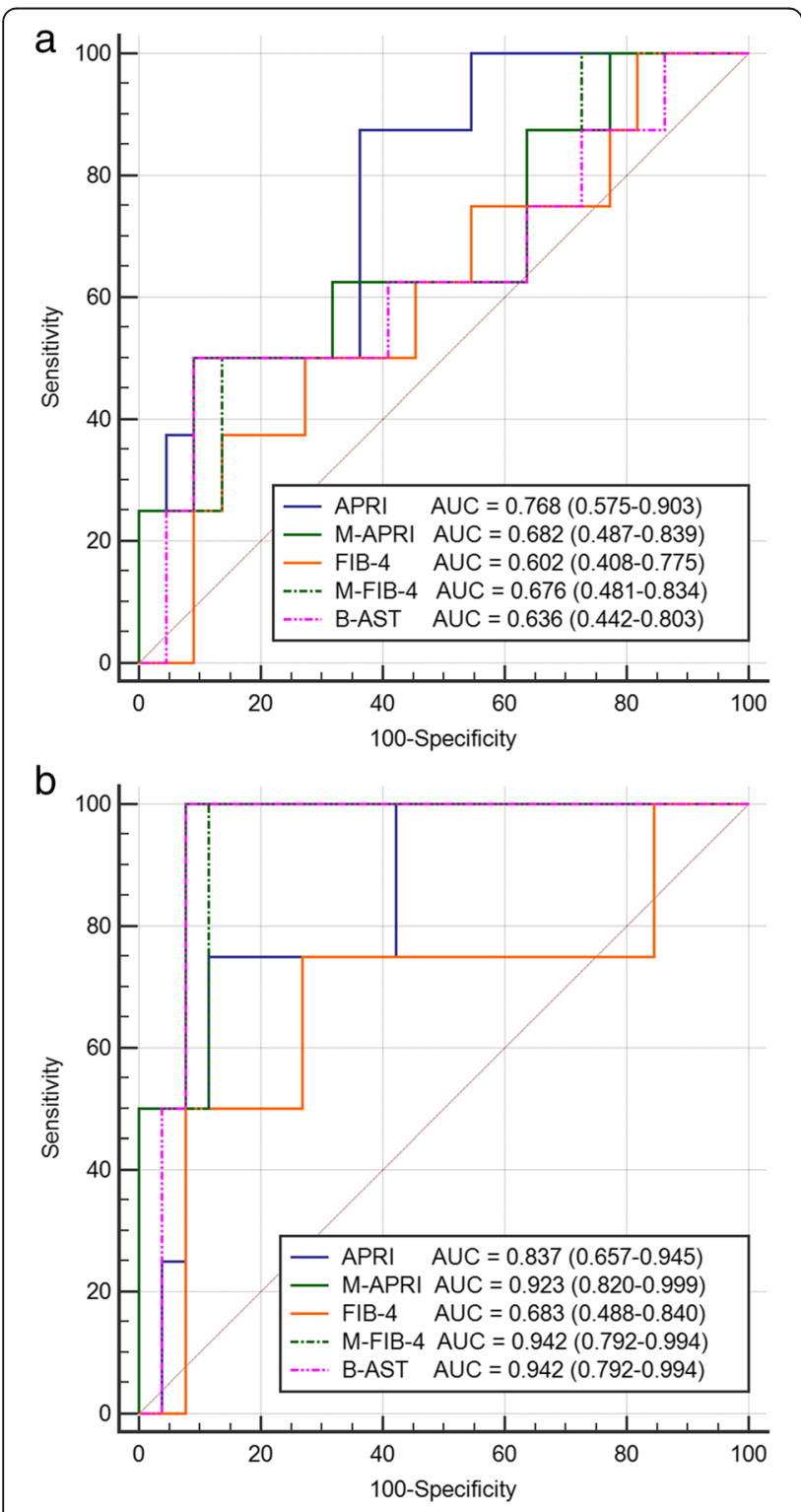

Fig. 2 Receiver operating characteristic (ROC) curves of the five noninvasive liver fibrosis tests for detecting steatosis. a for any steatosis. $\mathbf{b}$ for significant steatosis (> 33\% of hepatocytes affected)
$90 \%$ for advanced fibrosis, and FIB- 4 scores $>3.25$ had a $97 \%$ specificity and a positive predictive value of $65 \%$ for advanced fibrosis [6].

There are only limited published data on the use of these noninvasive methods in children, and, to our knowledge, none of these serum biomarkers have been fully validated in children with $\mathrm{CHC}$ to date. De Ledinghen et al. prospectively analyzed the feasibility of a liver stiffness measurement using FibroScan (elastography method) in 116 children with chronic liver diseases and compared the results with those of FibroTest (a commercial biomarker), the APRI and liver biopsy [4]. All three noninvasive methods correlated significantly with the METAVIR fibrosis score. The AUROCs for cirrhosis diagnoses were 0.88 for FibroScan, 0.73 for FibroTest, and 0.73 for the APRI. However, the cohort of patients in this study was heterogenic and included children with liver diseases of different etiologies.

In a study conducted in children and adolescents with chronic hepatitis B and C, the AUROC was 0.71 for identifying patients with fibrosis and 0.52 for identifying those with liver cirrhosis [14]. For children infected with $\mathrm{HCV}$, the AUROC was 0.75; it was more effective for vertically infected patients than for those infected via blood transfusion (1.00 vs. 0.53 ). The authors concluded that a validated noninvasive marker of fibrosis is needed for pediatric patients; however, the results of their study indicated that the APRI is not such a marker [14].

Our recent observations suggest that liver fibrosis in children with $\mathrm{CHC}$ is positively associated with the BMI $\mathrm{z}$-score. In both univariate and multivariate analyses, the BMI $\mathrm{z}$-score was found to be an independent predictor of fibrosis among our 42 pediatric patients with $\mathrm{CHC}$ $(p=0.03)$ [9]. Therefore, we proposed including the BMI z-score in the formulas of previously used biomarkers. The obtained results indicate that these modified tests perform better than the APRI and FIB-4 for predicting severe fibrosis. Additionally, we proposed a new simple biomarker, B-AST, that can easily detect liver fibrosis based on very simple parameters. B-AST, 
Table 4 Diagnostic performance of five non-invasive tests for determining significant steatosis (> 33\% of hepatocytes)

\begin{tabular}{llllll}
\hline Significant steatosis (> 33\% of hepatocytes) & & & & \\
\hline Test & APRI & M-APRI & FIB-4 & M-FIB-4 & B-AST \\
\hline AUROC (95\% Cl) & $0.837(0.657-0.945)$ & $0.923(0.820-0.999)$ & $0.683(0.488-0.840)$ & $0.942(0.792-0.994)$ & $0.942(0.792-0.994)$ \\
Cut-off & 0.656 & 0.577 & 0.216 & 0.179 & 92.82 \\
Sensitivity (95\% Cl) & $75(19.4-99.4)$ & $100(39.8-100)$ & $75(19.4-99.4)$ & $100(39.8-100)$ & $100(39.8-100)$ \\
Specificity (95\% Cl) & $88.46(69.8-97.6)$ & $92.3(74.9-99.1)$ & $73.1(52.2-88.4)$ & $88.46(69.8-97.6)$ & $92.3(74.9-99.1)$ \\
+ PV & $50.0(23.1-76.9)$ & $66.7(34.6-88.3)$ & $30.0(15.5-50.0)$ & $57.1(31.5-79.4)$ & $66.7(34.6-88.3)$ \\
- PV & $95.8(80.7-99.2)$ & 100 & $95.0(77.4-99.1)$ & 100 & 100
\end{tabular}

$A P R I$ aspartate-to-platelet ratio index, FIB-4 Fibrosis-4 index, M-APRI modified aspartate-to-platelet ratio index (BMI z-score $x$ APRI), M-FIB-4 modified Fibrosis-4 index (BMI z-score x FIB-4), B-AST BMI Z-score x AST, AUROC area under the receiver operating characteristic, $95 \% \mathrm{Cl} 95 \%$ confidence interval, $+P V$ positive predictive value, $-P V$ negative predictive value

with a cut-off of 92.8 , showed $71 \%$ sensitivity and $95 \%$ specificity for detecting significant fibrosis.

Several studies have demonstrated that liver steatosis in patients with $\mathrm{CHC}$ may have prognostic and metabolic implications [15-17]. In our recent study, moderate to severe steatosis was independently associated with the BMI z-score in a group of 48 patients with $\mathrm{CHC}$ $(p=0.02)$ [10]. In addition, it was found to be a predictor of advanced fibrosis in children with HCV infection; this was a unique finding because unlike in adults, no correlation between liver steatosis and fibrosis had previously been confirmed in pediatric patients with $\mathrm{CHC}$ [10]. Noninvasive markers of steatosis have been studied in patients with nonalcoholic fatty liver disease (NAFLD) but not in patients with CHC [18]. The results of our study suggest that biomarkers that include the BMI z-score perform excellently for diagnosing significant steatosis in children with CHC. B-AST had a very high sensitivity (100\%) and specificity (92\%) for predicting severe steatosis with a cut-off of 92.8. Considering that negative B-AST values excluded all patients with both significant fibrosis and significant steatosis, one may speculate that liver biopsy could be avoided in children with B-AST $<0$. In this study, 11/30 (37\%) of children had $\mathrm{B}-\mathrm{AST}<0$ and consequently would not require the liver biopsy.

Despite the novel and unique findings presented in this study, several issues should be considered as limitations. First, the small number of patients in the study group should be acknowledged, and the obtained results should be confirmed with other, larger cohorts of children. However, currently, liver biopsy is rarely performed in children; thus, the opportunity to compare noninvasive methods with histopathological assessment as a reference standard is lacking. Another important issue arose from the relatively mild liver disease observed in the histopathological evaluations: a low number of children presented with significant fibrosis, and no children presented with cirrhosis, which could lead to a spectrum bias. However, children with $\mathrm{CHC}$ usually present with mild liver disease, and cirrhosis is rarely observed [9]. Therefore, in most cases, distinguishing no or mild fibrosis from significant fibrosis would be most desirable for therapeutic decision-making.

\section{Conclusions}

In conclusion, the results of this study indicate that noninvasive biomarkers that include BMI z-scores in their formulas have a good to excellent performance for detecting significant liver disease. The novel simple B-AST serum test may be an inexpensive and widely available alternative to liver biopsy due to its high sensitivity and specificity for detecting significant liver fibrosis and steatosis. Continued research in this area in pediatric populations is needed to fully validate these noninvasive diagnostic tools for children with chronic liver diseases of different etiologies.

\section{Abbreviations}

ALT: Alanine aminotransferase; APRI: Aspartate aminotransferase-to-platelet ratio index; AST: Aspartate aminotransferase; AUROC: Area under the receiver operating characteristic; BMI z-score: Body mass index z-score; CHC: Chronic hepatitis C; Cl: Confidence interval; HCV: Hepatitis C virus; IQR: Interquartile ranges; PV: Predictive value; ROC: Receiver operating characteristic;

SD: Standard deviation; ULN: Upper limit of normal

\section{Acknowledgements}

Not applicable.

\section{Funding}

Not applicable.

\section{Availability of data and materials}

The datasets used and analyzed during the current study are available from the corresponding author upon reasonable request.

\section{Authors' contributions}

MPS performed the research, designed the research study, conducted the statistical analysis, and drafted the manuscript; MPS, BKM, MA, and MP collected and analyzed the data; MM contributed to the study design; BKM, $M A, M P$, and MM contributed to the interpretation of the data, and critically revised the manuscript; all authors read and approved the final manuscript.

\section{Competing interests}

The authors declare that they have no competing interests. 


\section{Consent for publication}

Not applicable.

\section{Ethics approval and consent to participate}

The investigation was concordant with the principles outlined in the Declaration of Helsinki and its amendments. Written informed consent was collected from all the patients and/or their parents/guardians before the biopsy examination. The local ethics committee at the Medical University of Warsaw, Poland, approved the study.

\section{Publisher's Note}

Springer Nature remains neutral with regard to jurisdictional claims in published maps and institutional affiliations.

Received: 3 March 2017 Accepted: 14 May 2017

Published online: 23 May 2017

\section{References}

1. Dezsofi A, Baumann U, Dhawan A, Durmaz O, Fischler B, Hadzic N, Hierro L, Lacaille F, McLin VA, Nobili V, et al. Liver biopsy in children: position paper of the ESPGHAN Hepatology committee. J Pediatr Gastroenterol Nutr. 2015;60(3):408-20

2. European Association for the Study of the Liver. EASL recommendations on treatment of hepatitis C 2016. J Hepatol. 2017;66(1):153-94.

3. Pokorska-Śpiewak M, Kowalik-Mikołajewska B, Aniszewska M, Pluta M, Marczyńska M. Is liver biopsy still needed in children with chronic viral hepatitis? World J Gastroenterol. 2015;21(42):12141-9.

4. de Ledinghen V, Le Bail B, Rebouissoux L, Fournier C, Foucher J, Miette V, Castera L, Sandrin L, Merrouche W, Lavrand F, et al. Liver stiffness measurement in children using FibroScan: feasibility study and comparison with Fibrotest, aspartate transaminase to platelets ratio index, and liver biopsy. J Pediatr Gastroenterol Nutr. 2007:45(4):443-50.

5. Castera L. Noninvasive methods to assess liver disease in patients with hepatitis B or C. Gastroenterology. 2012;142(6):1293-1302.e1294.

6. Sterling RK, Lissen E, Clumeck N, Sola R, Correa MC, Montaner J, S Sulkowski M, Torriani FJ, Dieterich DT, Thomas DL et al: Development of a simple noninvasive index to predict significant fibrosis in patients with HIV/HCV coinfection. Hepatology 2006, 43(6):1317-1325.

7. Wai CT, Greenson JK, Fontana RJ, Kalbfleisch JD, Marrero JA, Conjeevaram HS, Lok AS. A simple noninvasive index can predict both significant fibrosis and cirrhosis in patients with chronic hepatitis C. Hepatology. 2003;38(2):518-26.

8. Zeng DW, Dong J, Liu YR, Jiang JJ, Zhu YY. Noninvasive models for assessment of liver fibrosis in patients with chronic hepatitis B virus infection. World J Gastroenterol. 2016;22(29):6663-72.

9. Pokorska-Śpiewak M, Kowalik-Mikołajewska B, Aniszewska M, Pluta M, Walewska-Zielecka B, Marczyńska M. Determinants of liver disease progression in children with chronic hepatitis $C$ virus infection. Pol J Pathol. 2015;66(4):368-75.

10. Pokorska-Śpiewak M, Kowalik-Mikołajewska B, Aniszewska M, Pluta M, Walewska-Zielecka B, Marczyńska M. Liver steatosis in children with chronic hepatitis B and C: prevalence, predictors, and impact on disease progression. Medicine (Baltimore). 2017;96(3):e5832.

11. Bedossa P, Poynard T. An algorithm for the grading of activity in chronic hepatitis C. The METAVIR Cooperative Study Group. Hepatology. 1996;24(2):289-93

12. Panel AlHG. Hepatitis C guidance: AASLD-IDSA recommendations for testing, managing, and treating adults infected with hepatitis $C$ virus. Hepatology. 2015;62(3):932-54.

13. Lin ZH, Xin YN, Dong QJ, Wang Q, Jiang XJ, Zhan SH, Sun Y, Xuan SY. Performance of the aspartate aminotransferase-to-platelet ratio index for the staging of hepatitis C-related fibrosis: an updated meta-analysis. Hepatology. 2011;53(3):726-36.

14. McGoogan KE, Smith PB, Choi SS, Berman W, Jhaveri R. Performance of the AST-to-platelet ratio index as a noninvasive marker of fibrosis in pediatric patients with chronic viral hepatitis. J Pediatr Gastroenterol Nutr. 2010;50(3):344-6.

15. Adinolfi LE, Gambardella M, Andreana A, Tripodi MF, Utili R, Ruggiero G. Steatosis accelerates the progression of liver damage of chronic hepatitis $C$ patients and correlates with specific HCV genotype and visceral obesity. Hepatology. 2001;33(6):1358-64.
16. Bjornsson E, Angulo P. Hepatitis C and steatosis. Arch Med Res. 2007:38(6):621-7.

17. Guido M, Bortolotti F, Jara P, Giacomelli L, Fassan M, Hierro L, Nebbia G, Zancan L, Rugge M. Liver steatosis in children with chronic hepatitis C. Am J Gastroenterol. 2006;101(11):2611-5.

18. Papagianni M, Sofogianni A, Tziomalos K. Non-invasive methods for the diagnosis of nonalcoholic fatty liver disease. World J Hepatol. 2015;7(4):638-48.

\section{Submit your next manuscript to BioMed Central and we will help you at every step:}

- We accept pre-submission inquiries

- Our selector tool helps you to find the most relevant journal

- We provide round the clock customer support

- Convenient online submission

- Thorough peer review

- Inclusion in PubMed and all major indexing services

- Maximum visibility for your research

Submit your manuscript at www.biomedcentral.com/submit
) Biomed Central 